\title{
Strates
}

STRATES Matériaux pour la recherche en sciences sociales

$7 \mid 1993$

Témoins du monde : Bulgarie, identités chinoises, explorer l'île de France

\section{Lire : la clé du monde}

Récit d'une institutrice bigouden

\section{Léontine Cariou}

\section{(2) OpenEdition \\ Journals}

Édition électronique

URL : http://journals.openedition.org/strates/1181

DOI : 10.4000/strates. 1181

ISSN : $1777-5442$

Éditeur

Laboratoire Ladyss

Édition imprimée

Date de publication : 30 juin 1993

ISSN : 0768-8067

Référence électronique

Léontine Cariou, «Lire : la clé du monde », Strates [En ligne], 7 | 1993, mis en ligne le 20 décembre 2005, consulté le 08 septembre 2020. URL : http://journals.openedition.org/strates/1181 ; DOI : https://doi.org/10.4000/strates. 1181

Ce document a été généré automatiquement le 8 septembre 2020.

Tous droits réservés 


\title{
Lire : la clé du monde
}

\author{
Récit d'une institutrice bigouden ${ }^{1}$
}

\section{Léontine Cariou}

\section{NOTE DE L'ÉDITEUR}

Propos recueillis par Raymonde Ladefroux et Pernette Grandjean et présentés par Michèle Petit.

Les lettres déchiffrées

1 Pour mon père, ma mère, c'était quelqu'un, parce qu'elle était allée à l'école, elle savait lire, elle écrivait de sa belle écriture penchée, et mon père vénérait beaucoup ma mère de ce côté-là je crois, parce qu'il n'avait pas eu dans son enfance ce qu'elle avait eu. Pourtant, tous deux ont eu des enfances affreusement malheureuses : ma mère a perdu son père quand elle avait deux mois, et mon père était l'aîné de huit enfants : à douze ans, il était vacher. C'était une enfance épouvantable.

2 Ma mère, qui était née en 1884, avait été élevée chez les religieuses. Elle nous lisait la vie des saints - c'est à peu près tout ce qu'elle lisait - pas tous les jours, mais quelquefois le soir. Et mon père assistait, même si ça ne l'intéressait pas.

3 Il n'était jamais allé à l'école, et il était avide de savoir et de tout comprendre. Il avait appris à lire tout seul. Parce que pendant la guerre, ma mère lui écrivait et il avait un camarade, un prêtre, qui lui lisait ses lettres. Il lui lisait et relisait, si bien que mon père les connaissait par cœur. Il en avait à peu près six. Quand le prêtre a changé de secteur, mon père s'est retrouvé tout seul et il s'est dit: "Je ne vais tout de même pas encore demander à quelqu'un d'écrire pour moi ! » Alors il prenait les lettres de ma mère, il les étalait, il cherchait ses mots par comparaison, et il les dessinait. Ma mère nous a dit un jour: «La première lettre que j'ai reçu de votre père, je devinais, mais après il s'appliquait bien et moi, quand j'écrivais, je formais bien mes lettres pour qu'il puisse deviner les mots. Mais du moins, il y avait un contact entre nous. » Quand il est rentré de la guerre, il a appris à lire avec ses enfants, dans nos livres de lecture. 
Il était de tendance "gauche», il s'est abonné au «Populaire», et il avait son « Populaire » ostensiblement, il l'ouvrait devant tout le monde, c'était sa revanche... La lecture lui permettait de se tenir au courant des événements et il savait ce qu'il lisait, il comprenait très bien ce qu'il lisait.

L'école du diable

5 L'autre jour, je passais devant l'école des religieuses qui n'existe plus - c'est maintenant une colonie de vacances -, je rencontre une dame: «Ah!» me dit-elle, «voilà mon école ! » Puis, me regardant, elle ajoute : «C'est vrai que vous, vous n'avez pas pu aller à cette école...» (Comme si c'était un privilège!) «C'est vrai que votre père était cantonnier de commune, vous n'avez pas pu venir dans cette école. » J'ai répondu: «Jamais mon père n'aurait accepté que je vienne dans une école privée catholique alors qu'il y avait une école d'État!» Ma mère était pratiquante, mais jamais mon père n'aurait cédé sur cette chose, sous n'importe quelle influence. La personne qui m'a dit ça avait une dizaine d'années de moins que moi, la soixantaine...

6 Mon père était cantonnier, il pouvait sans craindre les foudres du clergé confier ses enfants à l'école laïque, l'école de l'État, puisqu'il était payé par cet État laïque. A l'époque, vous savez, chez nous, c'était la guerre contre l'école publique! A deux ans et demi j'ai donc été à la maternelle, à Plobannalec. L'institutrice était une cliente de ma mère qui était couturière, et elle a dit à ma mère : «Donnez-la moi ». J'ai eu droit, à deux ans et demi, de prendre place sur l'espèce de petit banc qu'on mettait sur le côté de la classe et de voir ces tableaux, avec une grande qui lisait B A, BA, etc. Et j'ai appris à lire petit à petit. A quatre ans, je savais lire.

7 Et le prêtre nous parlait en chaire (parce que ma mère allait à la messe tous les dimanches) de "l'école du diable ». Je ne comprenais pas très bien ce que c'était, et un jour, un peu avant l'ouverture de la classe, après midi, je suis allée m'asseoir sur le seuil de la porte de l'école et j'ai entendu du bruit - je pense que c'était l'institutrice qui déplaçait quelque chose. Je suis revenue en hurlant à la maison en disant à ma mère en breton, bien sûr, je ne parlais que breton -: «J'ai entendu le diable!» Et je ne voulais plus retourner à l'école ! Il a fallu que ma mère me dise que ce n'était pas vrai...

8 La classe était en français, mais ma mère qui avait donc été à l'école, nous avait appris un petit peu le français à la maison avant qu'on aille à l'école, pour qu'on ne soit pas traumatisées.

9 A cette époque, la qualité de l'enseignement n'était pas la même dans les deux écoles, publique et religieuse. Même si les institutrices n'avaient que le BEPC. A l'école religieuse, il n'y avait pas grand-chose : c'était des religieuses qui avaient un peu de savoir... Vous savez, ce n'est pas difficile d'apprendre à lire. N'importe qui peut apprendre à lire, mais il y a «apprendre à lire » et «apprendre à lire »: c'est quand même différent. Le mécanisme peut être appris, mais il y a aussi apprendre à l'enfant à aimer lire, toute la différence est là, qu'un enfant se dise : « Oh ! Chic ! Je vais apprendre à lire ! » Tout est là, c'est de faire aimer lire, découvrir, dès le départ, donner ce goût de la découverte et d'aller très loin pour en savoir beaucoup plus. Et même maintenant qu'on croit savoir beaucoup de choses, on ne sait rien.

10 Mes premières lectures, c'était d'abord mon tableau de lecture de l'école, puis les livres de lecture de mes sœurs. Je crois que c'était la méthode Boscher à l'époque. J'étais avide, j'étais jalouse de les voir lire. Ma mère disait toujours qu'il fallait qu'on aille me promener pendant que les autres lisaient, parce que moi je ne savais pas et que je 
perturbais. Mais j'avais une sorte de ténacité de savoir et d'apprendre. Et puis après j'ai eu une nouvelle institutrice qui avait une fille un peu plus jeune que moi et qui était abonnée à Lisette. Alors je faisais des pieds et des mains, pensez, pour être l'amie première, et $j$ 'avais Lisette toutes les semaines.

Des conteurs à la télé

11 Mais vous savez, pour les économies de lumière, nous avions le droit de lire à la nuit tombante, entre chien et loup, le nez collé à la vitre. Pendant que ma mère préparait le repas, il y avait la petite lampe pigeon, pour les repas on avait la grosse lampe. Quand on allumait la grosse lampe, on avait aussi le droit de lire un peu, mais que voulez-vous, quand les gens arrivaient pour la veillée, les hommes parlaient en fumant, les femmes tricotaient ou cousaient et nous, on ne pouvait pas lire.

Il y avait les veillées, pas tous les jours, mais enfin souvent, chez l'un, chez l'autre. Et pendant longtemps, ça a été chez nous, parce que nous avons été les premiers à avoir l'électricité. Alors ça a été un événement! Le jour où mon père a allumé, les gens du quartier y étaient, et il y a eu un : « Oh !»

13 Mais je n'ai pas connu beaucoup de conteurs ici : c'était plutôt dans le centre de la Bretagne, dans la Montagne noire, les monts d'Arrée, des gens qui étaient encore plus isolés que nous. Peut-être au temps de ma petite jeunesse y en avait-il davantage, je ne me souviens plus.

14 C'était des conteurs occasionnels. Mon père était un conteur, il inventait. J'ai eu l'occasion de voir Pierre Jakez Hélias pour qu'il me dédicace son bouquin. Il m'a dit: «C'est dommage que vous ne me l'ayez pas fait connaître, parce que je l'aurais fait passer à l'émission en breton ». Il allait là-bas, au café, pour avoir sa cour. Et il racontait des histoires...

Les contes, c'était oral, et ça remplaçait la lecture. Par la suite, on tâchait de retrouver les émotions que l'on avait en écoutant ces contes-là, et on a essayé de les retrouver dans la lecture. A partir du moment où les veillées se sont estompées, on a été en manque et on a cherché dans la lecture autre chose. Oui parce que les veillées ont duré jusqu'à la seconde guerre, par là. Après la guerre, c'était fini. A ce moment là, il y a eu l'électricité partout et il y a eu les postes de TSF. Et Pierre Jakez Hélias a été celui qui a révélé ce besoin de culture chez les gens. Vous savez qu'on lui doit beaucoup ? Il y avait une émission en breton, il y avait deux compères...

16 J'avais acheté un petit poste de radio, je l'ai laissé à mes parents en 46. Eh bien, tous les dimanches, mon père allait chercher ses vieux copains pour écouter l'émission de Pierre Jakez Hélias. Et cette réunion, ça remplaçait la veillée, si vous voulez. Ça se passait à trois heures de l'après-midi le dimanche. Le fait que j'ai pu gagner un peu d'argent de par ma situation m'a permis de donner à mes parents ce qui leur a beaucoup manqué si longtemps, cette partie culturelle: "C'est pas possible, tu nous laisses ta boîte rouge !» me disait mon père. Quand je me suis acheté un poste de télé, j'en ai acheté deux le même jour, et je l'ai fait installer chez mes parents. Mon père était dans le jardin. Je lui ai dit : « Tu viens, il y a un cadeau pour toi, là. » Alors il est venu et a dit en breton : « Le râteau! » (l'antenne) Ah! Il avait plein de larmes dans les yeux, il n'en revenait pas, tant d'émotion! C'était le summum, là il y avait des copains qui venaient... et ça lui a ouvert le monde.

17 C'était une époque où la télé ouvrait beaucoup plus sur le monde que maintenant, où elle est destructrice et négative, à mon point de vue. Les très bonnes émissions sont 
très tard le soir, alors c'est inabordable pour les gens qui travaillent, et pour ceux qui, à leur retraite, ont envie de se cultiver un peu, d'en savoir un peu plus. Et tout le monde n'a pas de magnétoscope... Moi je n'en veux pas parce que je ne veux pas me plier à cet ordre de choses. Et puis ça m'enlèverait aussi du temps pour lire, parce que je lis facilement de neuf heures à dix heures et demie.

Lectures de pension

Dans mon enfance, il y avait peu de livres dans la région. Il n'était pas question d'en acheter, parce qu'il n'y avait pas d'argent. Mais à l'école, il y en avait peut-être une dizaine.

Après, à douze ans, je suis allée à Quimperlé, j'étais en pension, hélas ! Mais là aussi, j'ai lu, oh la la ! Il y avait une bibliothèque... et puis un prof de français qui m'a beaucoup impressionnée, qui était aussi prof de musique, et qui m'a fait découvrir le monde de la musique! Parce que j'étais coupée de tout ça... Il n'y avait que les cantiques à l'église, la musique religieuse. Autrement... on allait dans les fermes où ils refaisaient les aires nouvelles, qu'ils remplissaient avec de la terre : les gens venaient là danser la gavotte ; il y avait des binious, alors on y allait le dimanche après-midi danser pour tasser cette terre. On était toujours pour de l'utilité : on dansait, mais c'était pour quelque chose de très utile. Vous savez, les binious sur les tonneaux, c'était réel. On ne prenait pas le temps d'installer des estrades...

Je suis donc restée en pension jusqu'au Brevet supérieur. J'ai lu tout ce qui me tombait sous la main. Les « Claudine », bien sûr, et les « Malheurs de Sophie »! Et puis... j'aimais beaucoup les bouquins d'exploration : toujours cette idée d'aller loin.

A l'époque, je ne me souviens pas d'avoir aimé vraiment les romans d'amour. Je ne sais pas, j'étais peut-être pudique, sans doute, je tenais ça de mon éducation qui était tellement stricte. Il y a des choses que je n'osais pas approcher... Mais on a lu «L'amant de Lady Chaterley » sous les pupitres... Moi j'étais très naïve, je ne sais pas si j'avais très bien compris. Je l'ai lu depuis, vous pensez bien...

Vous savez, ce que je lisais beaucoup aussi - ça va peut-être vous étonner - c'est le dictionnaire... J'aimais ça. Et j'ai eu beaucoup de mal en français du fait qu'il me manquait du vocabulaire. Je parlais breton, et les tournures bretonnes ça a été une catastrophe et un souci énorme pour les profs de français: le mot "avec", par exemple. Vous ne connaissez pas l'expression, peut-être... Il y a une estivante à Pontl'Abbé le mois dernier qui demandait à une marchande de poisson : « Il est frais votre poisson, Madame? » «Oh oui » répond la marchande, «il a été avec moi toute la nuit dans la glace ». Ce qui signifie en réalité : « Je l'ai mis toute la nuit dans la glace » : c'est de la traduction littérale. Nos professeurs de français avaient énormément de mal avec des enfants qui n'avaient pratiquement jamais parlé le français. Il y a des enfants qui venaient à l'école et qui ne connaissaient pas un seul mot de français. Quand j'étais institutrice, j'ai eu un petit qui, après ma petite histoire de morale, m'a dit en breton : «Maintenant dis-le moi en breton, parce que je n'ai rien compris ». Alors comme c'était au $\mathrm{CP}$, à la récréation je lui ai raconté mon histoire. Eh bien, à la fin de l'année, il lisait, il parlait français, parce qu'un enfant ça apprend très vite et beaucoup plus facilement qu'une grande personne.

23 Les philosophes, je les ai lus d'abord par obligation. Je mentirais si je vous disais que je me jetais dessus pour les avaler. Mais je les ai lus et j'y ai pris du plaisir, à la longue. C'est plus tard, avec plus de maturité, que je les ai lus. Je trouve qu'on fait la philosophie à des enfants trop jeunes. Moi j'étais attachée à ma directrice à Quimperlé, 
qui venait nous expliquer la musique, parce que nous étions une petite dizaine d'avides de musique. Nous avions le privilège d'aller prendre le thé chez elle. Et elle nous expliquait certains morceaux que nous ne comprenions pas. Elle était aussi prof de philo. Alors, parce que je l'aimais beaucoup, pour lui faire plaisir je m'accrochais à la philo, mais au début je trouvais ça un peu rébarbatif. Mais c'était quand même des cours de discussion, parce qu'elle nous faisait beaucoup parler. C'est sans doute une amorce d'une recherche de philosophie. J'ai apprécié plus tard, quand j'ai eu une expérience de vie, et c'est essentiel pour pouvoir approcher la philosophie. Je dis approcher, parce qu'il faut être modeste. J'ai réalisé la chance que j'ai eu dans ma vie de pouvoir lire tout ce que j'ai lu, de pouvoir connaître tout ce que j'ai connu, alors que sans une chance de départ, je restais là, j'allais travailler à l'usine et je restais à peu près au même niveau.

Découvrir

Quand j'ai travaillé, j'ai continué à lire. J'ai trouvé des bibliothèques, une grande à Kérity, et à Pont-l'Abbé, et puis j'ai acheté beaucoup de bouquins. J'ai été abonnée à la Guilde du Livre qui m'a fourni des bouquins magnifiques. On recevait un petit opuscule tous les mois, on était obligés de prendre un livre tous les deux mois, je crois. C'est là que je me suis fait ma bibliothèque, avec ce guide qui était vraiment très sérieux, très compétent, de très beaux livres, tout ce qui paraissait. Et surtout de qualité.

Actuellement, les livres par souscription, c'est affreux. C'est la vente à tout prix, du beau livre, qui a une belle couverture, un beau papier, mais il n'y a rien dedans. Alors que la Guilde du Livre, c'était une sélection de livres de presque tous les pays du monde, puisque c'était imprimé à Lausanne.

Moi j'aime bien connaître la vie des gens dans les différents pays. C'est le genre de livres que j'aime. Me sentir chez eux, avec eux, la vie de tous les jours, plus que le livre roman.

En ce qui concerne la littérature d'autres pays... je ne connais pas beaucoup la littérature sud-américaine, je n'en ai pas beaucoup lu. J'ai lu «La baie de tous les saints ", de Jorge Amado. Mais la littérature russe, j'en ai lu énormément, tout ce qu'on peut lire, c'est classique. J'ai trouvé ça beau et triste à la fois, c'est très prenant. C'est beau, et puis c'est terrible. J'ai beaucoup aimé. Qu'est-ce que j'ai aimé d'autre, en littérature étrangère? Mishima, j'ai adoré, je le trouve extraordinaire. J'ai ses œuvres en " poches», je les trouve magnifiques! C'est de la délicatesse, des fleurs japonaises, c'est de la soie.

Quant aux livres historiques... il y a tous les livres qui ont été écrits sur la Résistance, après la guerre. Là, que voulez-vous, moi j'ai sauté dessus, tout ce qui a paru à ce moment là, parce que c'était d'intérêt national, j'avais vécu ça aussi de près...

Ou sur la Révolution. J'ai lu le Condorcet des Badinter : alors quand on a lu ça, on a même une amertume qui vous reste, une grande amertume - voyez, la lecture donne des joies, de grandes joies, de grands plaisirs, et vous avez des amertumes aussi... On en est arrivé là après que cet homme... Après tout ce qu'il a dit... Comment ça se fait que l'humanité ait trahi cette chose-là ! Je trouve formidable que les Badinter aient donné ce soleil au public!

Aimer lire, c'est essentiel. On s'enrichit sur le plan intellectuel, on s'enrichit sur le plan affectif, on s'enrichit sur le plan émotionnel, tout. C'est une compréhension et une découverte. Parce qu'on ne lit pas que des romans, on lit autre chose ici. Je lis un peu 
tout ce qui me tombe sous la main. Il y a tout dans le fait d'aimer lire. Vous découvrez sur le plan littéraire, scientifique, géographique. Moi j'adore les atlas! Quand j'ai une heure devant moi, le soir, je prends un atlas, et je voyage, et je rêve, je rêve. Et maintenant, avec tout ce qui se passe en URSS, je regarde, là, et j'ai parfois du mal à lire le nom de ces États qu'on ne connaissait pas ou très peu il y a quelques années. Ça aussi, c'est de la lecture!

On ne peut pas apprendre sans effort. Et je pense qu'un enfant qui aime apprendre demande des choses difficiles. Un enfant est heureux quand il a dominé un effort. J'ai fait dix-sept ans de CP, j'ai enseigné la lecture pendant dix-sept ans. J'avais une méthode de lecture analytique. Je faisais ma méthode jusque vers le mois de février, et ensuite, je donnais un livre de lecture courante aux bons, et je repartais avec ma méthode avec les autres. A la fin de l'année, tout le paquet passait. Mais demandez ça maintenant... En fin d'année, les enfants du deuxième groupe avaient le livre de lecture courante et les meilleurs avaient une petite bibliothèque, et ils lisaient leurs bouquins pendant que je m'occupais des autres. Mais on n'avait le droit de changer de bouquin que quand on savait ce qu'on avait lu. Alors il y avait une séance qui se passait vers les quatre heures et demi (l'école finissait à cinq heures). Une gamine me disait: «Maîtresse, j'ai fini mon livre ». Je disais: "Viens me dire ce que tu as lu». Et elle s'asseyait à ma place. C'était un grand honneur, vous savez! Et elle racontait ce qu'elle avait lu. Quand je voyais qu'elle avait vraiment lu, et compris surtout, alors elle allait prendre un autre livre. Les autres lui posaient des questions. Je pense que les gosses qui sortaient de chez moi, ils avaient un bon niveau de lecture, ils savaient ce qu'ils lisaient, ils comprenaient ce qu'ils lisaient et ils avaient envie d'en savoir plus. Moi j'ai eu tellement envie de savoir beaucoup de choses, d'aller très loin, de connaître beaucoup de choses sur tout! Quand j'étais gamine, je voulais tout, tout savoir. Mon père était comme ça. C'était une avidité à la base.

Acheter des livres

32 Je viens de me payer les Pagnol dans une très belle édition. C'est une petite folie, mais je me suis dit : «Ça, c'est mon cadeau à moi, pour mon amour de la lecture. » Je me suis payé des Pagnol magnifiques! Et « 1789 » aussi. Ça, c'est la révolution, et ça, c'est mon enfance!

Mes livres reliés, je les ai mis ici... pour qu'on les voit. Mais ne vous inquiétez pas, j'ai des « poches » là-haut, dans les deux chambres il y a des " poches » partout.

Les livres sont très chers. J'achète une dizaine de bouquins par an peut-être, dans une librairie, à Pont-l'Abbé. Mais je n'achète pas aveuglément, j'achète sur les conseils de gens... J'ai acheté beaucoup à ce Club du Livre de Genève, mais quand ça s'est arrêté, j'ai eu une période vide, parce que je ne savais plus où m'adresser. Là, le robinet était constamment ouvert. Quand il s'est fermé, il m'a fallu un temps. Je n'avais plus la source, la facilité tous les mois d'avoir quatre livres au choix avec des résumés. Alors j'ai été un petit peu perturbée pendant un certain temps. Et puis je me suis quand même dit: «Il faut y aller ». Et j'ai trouvé dans des revues, ou à la radio, parce que j'écoute beaucoup France-Culture. J'ai aussi une amie qui est abonnée au Nouvel Observateur, et je trouve qu'ils ont de bonnes références.

Mais les prix littéraires, ça dépend. Je me documente avant, je lis pas mal d'articles, mais je ne saute pas dessus. Je trouve qu'il ne faut pas comme ça, a priori, dire : « Ils ont un prix, alors on achète ». C'est souvent du commerce. J'attends et on me dit: "Ah, c'est beau, il faut que tu achètes ça... » Alors ça dépend qui me le dit. Il y a un 
phénomène de mode. Et puis il faut que ce soit Gallimard ou un tel... Moi, je ne donne aucune importance à cette attribution de prix.

Quelquefois, ça m'arrive de relire. Et je trouve que dans la relecture d'un livre, on découvre encore des choses qui ont échappé à la première lecture, et c'est essentiel. Des descriptions... Il y a un livre de Pierre Jakez Hélias que j'ai relu, et j'y ai trouvé des choses qui m'avaient échappées. Et puis il y a des livres que j'ai relus, que j'avais aimés étant plus jeune, et je me suis dit : «Tu as lu ça, ou plutôt tu as donné tant d'importance à ça! »Ce n'était pas de la mauvaise lecture, je ne pense pas, mais je me suis dit par la suite qu'il y avait mieux. C'est adapté à l'âge.

Il y a aussi des gens qu'on a envie de lire, parce qu'on en parle tellement. Et puis... Vous avez lu « Un certain sourire » de Françoise Sagan? Je l'ai lu, eh bien je ne l'ai lu qu'une fois, ça m'a suffi, parce que je me suis dit: «C'est plat!» Ça m'étonne, l'importance qu'on lui donne.

Les échanges de lecture, c'est avec ma famille, c'est tout, et des amis. J'ai des amis qui lisent. Et puis l'été, des jeunes de ma famille viennent chez moi et me disent : « On peut prendre un livre? » J'ai ma sœur qui a quatre-vingt deux ans et qui, elle aussi, a un amour de la lecture. Elle lit énormément. Là, elle vient de me donner les quatre bouquins de Michelet, " Des grives aux loups $»^{2}$... On ne lui en avait offert que deux, et elle en a acheté un autre, puis le dernier. Mais le dernier est moins bien, on sent que ça s'effiloche, on sent qu'il s'accroche...

Je ne compte pas les livres que je lis par semaine, parce que quand il fait beau, je sors beaucoup, avec des amis, je fais beaucoup de marche, je jardine. J'ai donc moins de temps pour la lecture en été. La nature... c'est le plus beau des livres! Encore faut-il savoir le lire... avec ses yeux, avec ses oreilles, avec tous ses sens. Les livres aussi, d'une certaine façon... Il faut lire, relire, revenir en arrière.

Donner l'envie de lire

Je ne sais pas pourquoi, je ne vais plus beaucoup dans les bibliothèques. Il y a à Pontl'Abbé une bibliothèque très bien. J'ai des amis qui y vont et qui me disent : « Pourquoi n'y vas-tu pas ? » Mais pour l'instant, j'ai encore à lire. J'ai tort de ne plus y aller... Par laxisme, peut-être, parce que j'ai des choses à lire sous la main, je pique un livre ici, un livre là qu'on me prête, je crois que c'est ça. Je crois que je trouverais dans une bibliothèque - à Pont-l'Abbé ou ailleurs - à peu près tout ce que je cherche, j'en suis persuadée, mais c'est moi qui ne fais pas l'effort.

41 Il y a plus de gens qu'on ne pense qui vont à cette bibliothèque. Et je connais des gens... je n'aurais pas cru, qu'est-ce qu'ils achètent, qu'est-ce qu'ils prennent! Beaucoup d'estivants vont aussi à cette bibliothèque.

42 Les bibliothèques, c'est un grand problème. Je pense que la province est toujours parent pauvre. On n'a rien, et tout est centralisé sur Paris; Paris, c'est Paris. Jack Lang a promis pas mal de choses, mais on n'est pas encore arrivés à donner l'équivalence en province.

43 Les tournées de bibliobus en prêt direct... il faudrait tout d'abord qu'il y ait de la qualité dans cet apport de livres, qu'on ne nous apporte pas n'importe quoi. Et que ce soit par le biais de l'école, par le biais d'organismes qui pourraient se charger d'éduquer un peu les jeunes, de leur apprendre, de les mettre sur la voie, ne pas accepter le médiocre. Il faudrait des comités de lecture, des spécialistes, des gens qui s'y connaissent, et qui donneraient des livres de qualité aux enfants, qui seraient capables de guider un peu. Il 
ne suffit pas qu'il y ait une camionnette qui vienne apporter plein de bouquins là, sur une place publique et qu'on dise aux gens : « Servez-vous ». Ça ne sert à rien du tout. Ce qui est malheureux, à l'heure actuelle, c'est que justement les jeunes, ils ne demandent rien, mais on ne leur donne pas non plus assez de qualité. C'est ça qui manque, on les laisse un peu lire n'importe quoi, des parents leur donnent de l'argent, mais personne ne les conseille.

Et aussi, arrivés à la retraite, il y a des gens qui aimeraient peut-être lire et qui ne lisent jamais rien. C'est une grande tristesse, vous savez, des gens qui lisent leur Télégramme de Brest de $\mathrm{A}$ à $\mathrm{Z}$, à longueur de journée... et il y en a beaucoup. Ma sœur lit, mais ses voisines disent: «Toi, tu aimes lire des choses comme ça... » Des choses comme ça! C'est le terme qu'elles emploient !

Oui, pour les bibliothèques, il faudrait des professionnels, bien sûr. Parce que le bénévolat n'est jamais que quelque chose d'aléatoire, de passager, un passe-temps, pour certains... Des gens qui partiront un jour ou l'autre et qui ne seront pas remplacés, qui ne sont pas exactement à leur place. Il faudrait des gens compétents pour essayer de relever le niveau intellectuel, et le niveau moral, parce que ça va de pair.

Où va un pays qui ne donne pas priorité à l'éducation, à la lecture, à l'acquisition des connaissances? Actuellement, où allons-nous? A la décadence. Je vais peut-être un peu loin, mais c'est ce que je pense, absolument. Pourquoi ferme-t-on les yeux devant les jeunes? C'est de la démission totale. On est dans un pays où on fait semblant de s'occuper des jeunes : on va faire ceci, on va débloquer des crédits pour cela... Et ça ne vient jamais. Toutefois, il y a une commune, ici, au Guilvinec, qui a une Maison de la Culture, je ne sais pas exactement qui s'en occupe, mais vous pouvez être sûres que c'est très bien ce qu'ils font. Je vous parle de cet exemple que je connais : ma sœur habite près de cette Maison de la Culture. Cet hiver, ils ont fait une exposition de photos de première qualité. Je crois qu'ils ont un club de photo - ça occupe les jeunes aussi. Il y a un club de lecture, une bibliothèque importante, ils font des expositions, ils font venir des gens de Paris - et pas n'importe qui -, il y a des discussions, des chanteurs aussi, parce qu'il faut un peu de tout, des spectacles de qualité. Je pense que c'est la municipalité qui la gère.

Je pense qu'on ne peut rien faire si ça ne part pas de la municipalité même. C'est localement qu'il faut donner aux gens l'envie de lire et après, si on voit que ça marche, on fait des appels aux finances du gouvernement. Mais il faut que ça vienne de la base. La lecture démodée?

Et il faudrait beaucoup pour soulever la population, hélas, parce que je crois que c'est assez amorphe, il y a une dispersion, il y a autre chose qui semble prioritaire actuellement pour les gens que la lecture ou l'envie de se cultiver. C'est démodé, ça! C'est l'impression que j'ai... Pourtant il y a beaucoup de choses faites, mais qui y va?

$49 \mathrm{Au}$ Guilvinec, les estivants et une partie de la population vont à cette Maison de la Culture, quand il y a des nouveautés. Moi je vous dirai que je ne veux plus aller à des soupers marins, parce que ça, c'est pour les touristes. Mais il $\mathrm{y}$ a des expositions où les gens du pays ne vont pas, parce que ça ne les intéresse pas, ils ne sont pas accrochés.

Ils ont évolué comme tous les Français : ce n'est plus le cocon familial, c'est la famille éclatée ; c'est les copains, on va à la plage, on va en boîte... Les festivités régionales, ils y vont les jeunes marins, mais pour s'amuser, pour être avec les copains. Je ne pense pas qu'il y ait une recherche de racines... Ce n'est pas pire ici qu'ailleurs, le ton est le même. 
Et puis nous sommes dans une région riche, où les gens ont beaucoup d'argent. Le pêcheur d'ici est dépensier, mais pour la parade.

51 Il ne faut toutefois pas généraliser, parce que vous avez chez les pêcheurs des gens qui ont des niveaux de culture très élevés. Là où il y a eu un problème d'argent facile, ça $\mathrm{a}$ été dans les années soixante-soixante dix, qui a correspondu à une chute de l'agriculture. Et maintenant vous avez des agriculteurs qui se sont recyclés, ils ont fait des bateaux, qui ont aujourd'hui des tableaux de bord très sophistiqués... Il y a des marins très cultivés parmi les jeunes, de trente-trente cinq ans, qui lisent. Ils sont capables de suivre les débats à la télé, ils ont un autre niveau que celui des gens des années cinquante, qui allaient là parce qu'ils ne pouvaient pas aller ailleurs. Il n'y avait que la mer pour les prendre... Tandis que maintenant, ils vont vers la mer avec déjà un bon bagage au départ, et c'est pour ça qu'il y a maintenant cette pêche lointaine... Et je connais des marins qui ne lisent pas que des choses techniques... Beaucoup de marins s'occupent de l'éducation de leurs enfants. Il y a un grand pas de fait de ce côté là, un désir de promotion. Mais ça n'est pas encore au stade de la culture.

52 Dans ma famille, le désir de culture, c'était une soif. La promotion sociale allait de pair avec le bonheur en famille. C'était une promotion sociale qui allait dans le sens d'une reconnaissance. D'ailleurs, les gens riches que j'ai connus dans mon enfance n'étalaient pas leur richesse. Le paysan bigouden qui était très riche n'écrasait pas les autres. Les femmes s'habillaient comme tout le monde et les hommes allaient à la messe du dimanche habillés comme tout le monde. Ils avaient des maisons un peu plus cossues, bien sûr. Mais il n'y avait pas un étalage comme il y a aujourd'hui où il faut avoir une belle maison, une belle voiture. Le cheval d'orgueil, ça existe toujours !

Et l'amour de la Bretagne! Moi, je suis fière d'être Bretonne et je garde un respect et une vérité de mes origines, si pauvres qu'aient été mes parents. Heureusement, depuis il y a eu un progrès des conditions de vie matérielles, mais qui n'a pas apporté ce qu'il aurait dû apporter.

54 J'ai toujours lu dans ma vie. Et à la retraite, évidemment, ça m'a beaucoup aidée. Parce que pour moi, la retraite, ça a été un choc! La lecture a donc été une source d'apaisement. Déjà quand j'ai quitté le CP pour le CE2, je regrettais de ne plus pouvoir apprendre à lire aux petits : c'était une richesse, une joie intense ! J'ai eu une profession qui a été un summum dans ma vie. Le mot « instituteur » est un mot magnifique ! Parce qu'on est à la base, au départ. Si vous loupez le départ, vous pouvez louper beaucoup de choses dans la vie des enfants.

Avant l'instituteur était mal payé, mais il avait une fonction noble, et puis il avait un logement de fonction... Mais cette noblesse de la fonction n'existe plus, sauf pour quelques parents... C'est un problème social : l'instituteur est mal payé, et de ce fait il ne vaut rien ou presque. Voilà, c'est une question de monnaie, c'est tout. Ce qui est affreux actuellement, c'est l'emprise mal utilisée des parents d'élèves qui veulent se substituer à l'instituteur pour la manière d'enseigner. Si l'enfant ne travaille pas bien, ce n'est jamais l'enfant qui ne comprend pas, c'est le maître qui ne vaut rien, et ça, c'est dramatique. Il y a beaucoup de parents qui disent à l'enfant: "Ton maître ne vaut rien. » Et ça joue sur la lecture... Et puis il y a des parents qui ne font pas lire leurs enfants. Les enfants à l'école lisent vingt minutes dans la journée. Si les parents ne font pas lire l'enfant... L'apprentissage de la lecture, c'est un automatisme, il faut lire, plus on lit plus l'image se grave, plus on acquiert rapidement la lecture. 
Il y a aussi des inégalités sociales. Il y a des enfants qui ont une bibliothèque à sept ans, qui sont dans un bain de lecture... Les petits livres de carton qu'on donne aux enfants dès leur plus jeune âge, qu'ils manipulent, c'est un bon commencement. Et il faut très jeune qu'un enfant apprenne à respecter un livre, parce que le livre, c'est une chose qu'il faut respecter... Moi je faisais la guerre aux livres cornés! Il fallait que le livre soit aussi propre à la fin de l'année qu'au début.

Quand je vois des jeunes affalés dans un couloir de train avec des livres tout salis, cornés, j'ai mal au cœur ! Ca me fait mal aussi quand je vois casser un livre. Un livre ça se feuillette, il ne faut pas casser un livre, j'ai horreur de ça.

La musique des mots

Mes livres, je les range... harmonieusement. Je suis assez fantaisiste pour le classement. Mais j'ai horreur qu'on ne me rende pas un livre.

9 Il y a des livres que je peux lire avec la musique, mais pas n'importe quelle musique... J'écoute Mozart, parce que Mozart me pénètre, Mozart fait partie de ma lecture, il fait partie de moi-même... Je ferais n'importe quoi avec Mozart, même lire, ça ne me dérange pas du tout. Mais s'il y a du bruit, je ne peux pas.

Je ne peux pas lire dans un train, ou alors je lis des revues, mais quelque chose de passionnant non. J'aurais honte vis-à-vis d'un grand écrivain de le lire dans un train, c'est une offense. Je lis des revues, mais pour « lire ", j'aime être auprès d'un feu de cheminée, auprès d'un bel âtre, c'est un bonheur! Je n'envie personne... Dans ma lecture, je reviens souvent en arrière, je copie des passages... Ah! je suis dingue, parce que quand je les ai copiés, je les vois, ils sont plus beaux écrits par ma main, je ne sais pas comment vous dire, mais c'est quelque chose de très pur, de très entier, et de très sensuel, dans le bon sens du terme, oui, j'ai une grande sensualité dans ma lecture.

61 J'arrive à un moment de ma vie où je vois moins bien, il me faut des lunettes pour lire, alors quand je lis des caractères très gros, c'est agréable. Mais la qualité des éditions, ça n'ajoute rien au texte, je lis aussi bien dans un livre broché que dans un livre de poche. C'est le sens, c'est le français, c'est la musique des mots, la musique de la phrase qui m'intéressent, plus que le papier.

Quand je me fais un cadeau, je ne lésine pas, c'est quelque chose d'important, quelque chose qui va au cœur. J'ai aussi des livres sur la Révolution, notre Révolution, que j'honore, dans un beau papier. C'est aussi pour mes descendants, pour qu'ils voient, parce que ça aussi, j'y pense. Je me dis : la bibliothèque que j'ai, eh bien les petits qui viennent derrière, ils diront: "Tiens, elle lisait ça.» J'y tiens, ça entre dans la continuité. Je veux qu'ils sachent. D'abord j'écris pour qu'ils trouvent un jour, pour qu'ils disent : "Tiens, on avait un arrière grand-père comme ci... » C'est un héritage que je veux leur laisser. Dans les bouquins, j'ai des anecdotes, des papiers que je glisse... Mais annoter un livre, ça, jamais! J'ai aussi des cahiers dans lesquels j'annote des belles phrases que j'ai trouvées dans des bouquins qui ne sont pas à moi. Avant de les rendre, je copie des passages, et puis je les ai en tête.

Je n'achète pas des livres que j'ai lus après les avoir empruntés. Je préfère prendre du nouveau, aller vers d'autres lectures, parce qu'il y a des choses qui viennent, il y a des gens qui travaillent pour nous, vous savez... 


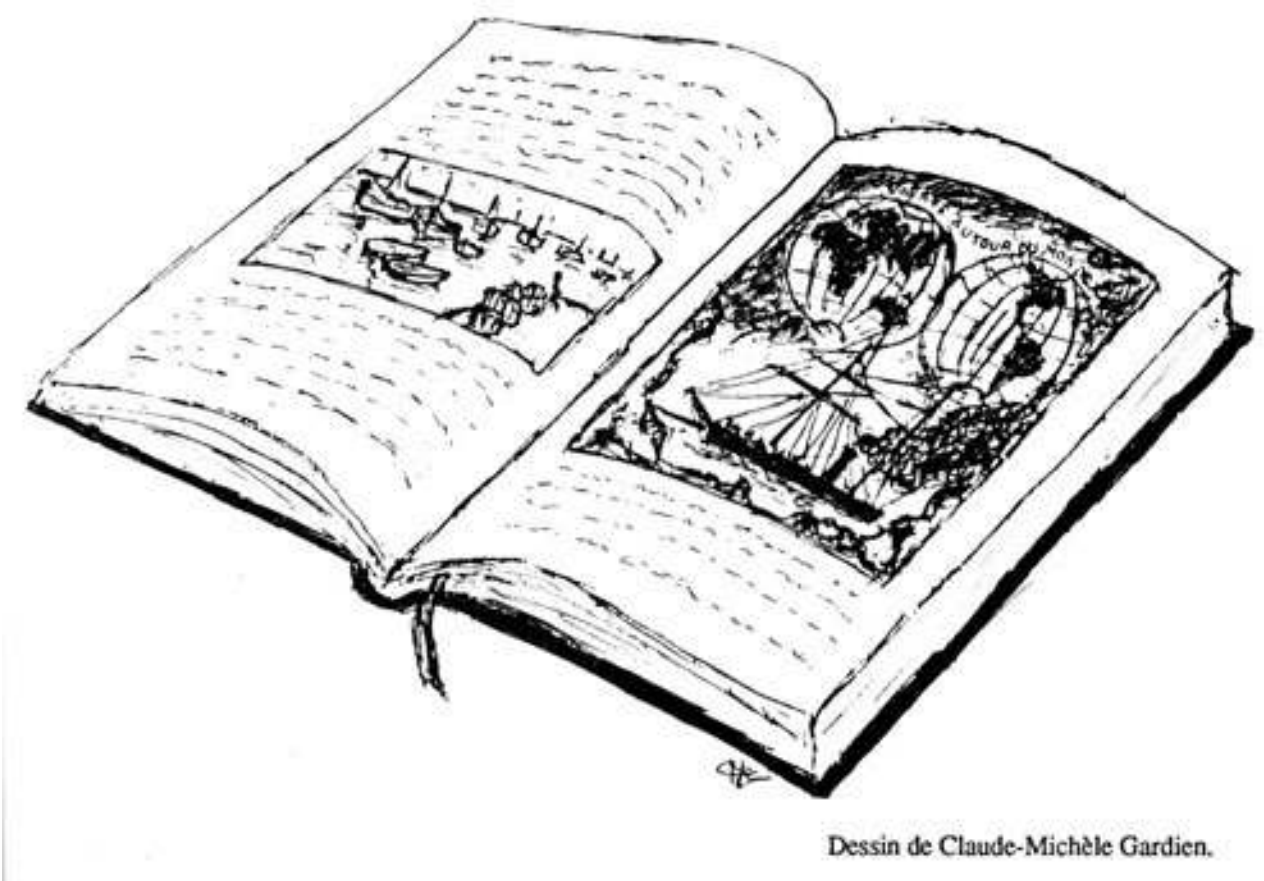

\section{NOTES}

1. Cet entretien a été réalisé en 1990 dans le cadre d'une recherche exploratoire sur $L a$ lecture en milieu rural. Financée par la Direction du Livre et de la Lecture du ministère de l'Éducation nationale et de la Culture, cette recherche a été menée par une équipe du laboratoire STRATES, en collaboration étroite avec le Service des études et de la recherche de la BPI du Centre Georges Pompidou. Nous remercions très vivement la BPI, et en particulier Monsieur Jacques Bourgain, Directeur de la BPI, et Madame Martine Chaudron, Chef du service des études et de la recherche, de nous avoir autorisés à publier ce récit. Les résultats de cette recherche sont publiés dans l'ouvrage Lecteurs en campagnes (Claude-Michèle Gardien, Raymonde Ladefroux et Michèle Petit), à paraître en automne 1993 aux Éditions de la Bibliothèque Publique d'Information du Centre Georges Pompidou (collection Études et recherches). Le montage de l'entretien a été réalisé par Michèle Petit.

2. L'un des tomes de la saga de Claude Michelet, publiée chez Laffont (NdE). 


\section{RÉSUMÉS}

Les pratiques culturelles ne sont pas seulement affaire de goût, d'accès au savoir, ou de distinction sociale, mais aussi d'ouverture sur des espaces plus ou moins larges : pour Léontine Cariou, originaire du pays bigouden, la lecture de livres a ouvert l'horizon sur d'autres continents, d'autres siècles. Cet entretien a été réalisé en 1990 dans le cadre d'une recherche exploratoire sur la lecture en milieu rural.

Reading : the key of the world. The story of a Bigouden schoolteacher.

Cultural practices are not only a matter of taste, access to knowledge, or social distinction, but also mediums for opening onto more or less wide worlds. For Léontine Cariou, a Brittany native from the Bigouden area, the reading of books has opened out new horizons over other continents and other centuries. This interview has been led in 1990 as part of an explorary research on reading in rural environments.

INDEX

Mots-clés : Pratiques culturelles, Lecture, Education, Récit de vie, Carnets de terrain, Espaces de référence, Pays bigouden

Keywords : Cultural practices, Bretagne, Reading, Life story, Field note-books, Spaces of reference, Brittany

\section{AUTEUR}

\section{LÉONTINE CARIOU}

A été institutrice et a vécu toute sa vie « à l'échelle » des livres. Donnant voix à la mémoire de son pays d'origine, le pays bigouden, elle habite aussi le monde, en citoyenne responsable et en poète. 